\title{
ChemComm
}

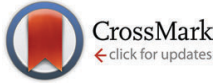

Cite this: Chem. Commun., 2015, 51,17225

Received 13th August 2015,

Accepted 23rd September 2015

DOI: $10.1039 / \mathrm{c} 5 \mathrm{cc} 06817 \mathrm{~g}$

www.rsc.org/chemcomm

\section{A rapid, sensitive colorimetric assay for the high- throughput screening of transaminases in liquid or solid-phase $\dagger$}

\author{
D. Baud, ${ }^{a}$ N. Ladkau, ${ }^{a}$ T. S. Moody, ${ }^{\text {b J. M. Ward }}{ }^{c}$ and H. C. Hailes ${ }^{{ }^{a}}$
}

\begin{abstract}
A new colorimetric method has been developed to screen transaminases using an inexpensive amine donor. The assay is sensitive, has a low level of background coloration, and can be used to identify and profile transaminase activities against aldehyde and ketone substrates in a high-throughput format. Significantly it is also amendable to solid phase colony screening.
\end{abstract}

Enantiomerically pure chiral amines are highly valuable building blocks in the pharmaceutical and fine chemical industries. ${ }^{1}$ However, established chemical synthetic routes for the preparation of such optically pure amines often require several reaction steps involving, for example, expensive transition metals and/or exhibit low yields because of poor regio- and stereoselectivity. ${ }^{2}$ The incorporation of more efficient and sustainable biotechnological alternatives and enzyme or whole-cell based bioprocesses, instead of synthetic chemistry transformations, has gained increased interest in recent years. ${ }^{3}$

Transaminases or aminotransferases (TAm, EC 2.6.1) are versatile biocatalysts for the efficient synthesis of enantiopure chiral amines. ${ }^{1 a, 4}$ There are six groups of transaminases, based upon amino acid sequence alignment, referred to as classes I to VI. Class III TAms have broader specificities and include the $\omega$-TAms which can accept structurally diverse aldehydes and prochiral ketones. ${ }^{4,5}$ TAms continue to attract significant attention due to wide ranging applications in the synthesis of $(R)$ and $(S)$ - amines and high levels of regio- and stereoselectivity that can be achieved. ${ }^{6}$ In addition, they are increasingly being reported for the preparation of bioactive and pharmaceutically important compounds. ${ }^{7}$ However, to establish an efficient transaminase-based bioprocess, problems such as substrate and product inhibition,

\footnotetext{
${ }^{a}$ Department of Chemistry, University College London, 20 Gordon Street, London WC1H OAJ, UK. E-mail: h.c.hailes@ucl.ac.uk; Tel: +44 (0)20 76797463

${ }^{b}$ Almac, Department of Biocatalysis and Isotope Chemistry, 20 Seagoe Industrial Estate, Craivagon, N. Ireland, BT63 5QD, UK

${ }^{c}$ Department of Biochemical Engineering, University College London, Gordon Street, London WC1H OAH, UK

$\dagger$ Electronic supplementary information (ESI) available: Characterisation data for 5 and assay protocols. See DOI: $10.1039 / \mathrm{c} 5 \mathrm{cc} 06817 \mathrm{~g}$
}

unfavorable reaction equilibria and tolerance to organic solvents for substrate solubilisation, must be overcome. Protein engineering in terms of semi-rational design and directed evolution presents a powerful strategy to tackle such issues, as demonstrated for example with the use of a TAm in the production of Januvia ${ }^{\circledR}$ (sitagliptin phosphate). ${ }^{7 a}$ To enable the rapid selection of either new productive native TAms or variants against particular substrates, fast, efficient, sensitive screening assays are required that are also low cost, unencumbered by intellectual property restrictions, and can be translated to a high-throughput (HT) mode of operation.

In recent years several methods for TAm screening have been established. $^{8}$ Assays for screening TAms against ketone and aldehyde acceptors include: the use of $\alpha$-methylbenzylamine (MBA) as an amine donor and acetophenone detection via HPLC or spectrophotometrically; ${ }^{9,10}$ a phenol red assay utilizing alanine as the donor and a combined lactate dehydrogenase and glucose dehydrogenase system (Scheme 1A), ${ }_{11}^{11}$ and a recent publication using ortho-xylylenediamine as amine donor, which on conversion to the aldehyde cyclizes to an isoindole and undergoes polymerization forming a black precipitate (Scheme 1B). ${ }^{12}$ Importantly, in this isoindole polymerization screen no additional enzymes are required reducing cost implications, and the assay can be applied in multiwell plates as well as in vivo on solid phase. However, a strong background reaction can be observed with some enzymes, and the high sensitivity precludes its use as a quantitative assay. ${ }^{12}$ Other methods have focused on amine donor screening using the acceptor pyruvate which generates alanine for detection via copper sulfate, ${ }^{13}$ or an amino acid oxidase coupled assay (Scheme 1C). ${ }^{14}$ In a related assay glyoxylate has been used as a donor with an amino acid oxidase coupled system with formation of a quinone imine dye. ${ }^{15}$ Although the enzyme-coupled assays offer high sensitivities, the need for additional enzymes (some are not readily available), and costly co-factors means that they are relatively expensive for larger screening projects. The copper sulfate assay exploits the formation of blue complexes but is an end-point assay, has less sensitivity, and is restricted to $\alpha$-keto acids as amine acceptors. ${ }^{13}$

Herein, we report a new colorimetric assay for the HT screening of TAms against aldehyde and ketone acceptors, 


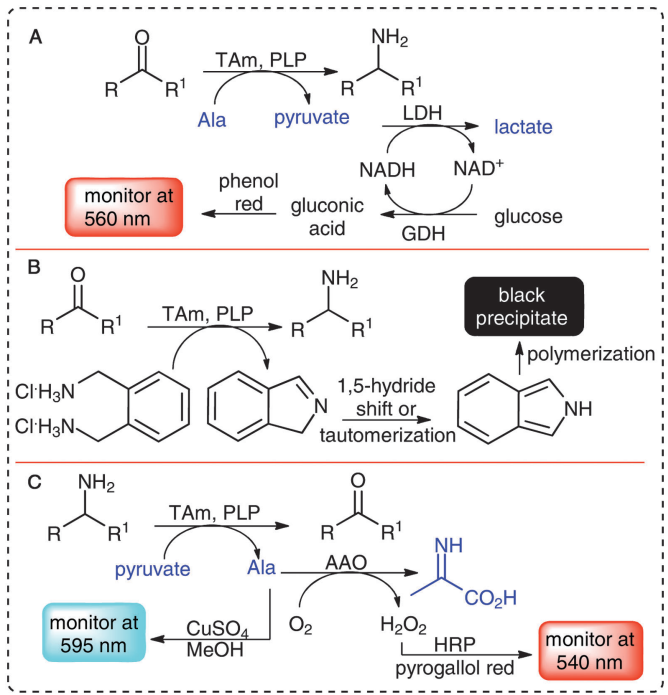

Scheme 1 Examples of colorimetric assays reported. Pyridoxal-5' ${ }^{\prime}$-phosphate (PLP). (A) Phenol red assay: lactate dehydrogenase (LDH) and glucose dehydrogenase (GDH). (B) ortho-Xylylenediamine assay. (C) Pyrogallol red and copper sulfate assay: aminoacid oxidase (AAO) and horseradish peroxidase (HRP).

which is applicable in liquid phase and for colony-based screening. This assay benefits from the use of an inexpensive amine donor, high sensitivity, as well as a low level of background reaction, and provides a quantitative read-out to approximately $30 \%$ conversion levels.

Initially, the study focussed on the use of commercially available 2-(4-nitrophenyl)ethan-1-amine $\mathbf{1}$ as an amine donor that when converted into the corresponding aldehyde $\mathbf{4}$ and subsequent basic work-up and deprotonation would give a highly conjugated structure with absorbance in the UV region. However, using $1(25 \mathrm{mM})$ for the transamination of benzaldehyde $2(10 \mathrm{mM})$ with Chromobacterium violaceum CV2025 $\omega$-TAm (CV-TAm), ${ }^{9}$ together with benzylamine 3 formation (67\% yield quantified via HPLC analysis after $18 \mathrm{~h}$ ) a red precipitate was also formed. After scale-up of the reaction and isolation of the red precipitate (purity $>85 \%, 35 \%$ yield, see ESI $\dagger$ ), the major component was identified as $\mathbf{5}$, indicating that the aldehyde 4 generated and the amino donor $\mathbf{1}$ formed an imine $\mathbf{6}$, which after tautomerization gave the conjugated red precipitate 5 (Scheme 2). Benzylamine 3, or more generally amines produced by TAms, can also react with $\mathbf{4}$ to form the corresponding enamine and contribute to the coloration observed. However $\mathbf{1}$ is present in higher equivalents so accounts for the major enamine formed.

To investigate the sensitivity of the assay, the level of coloration and conversion obtained was correlated in the CV-TAm reaction of 1 and 2 , which were applied in ratios of $1(25 \mathrm{mM}): 2(10 \mathrm{mM})$ and $1(12.5 \mathrm{mM}): 2(5 \mathrm{mM})$. The assay demonstrated a high sensitivity giving a visible colour change at conversion levels of only $2 \%$ (12.5 $\mathrm{mM} 1$ and $5 \mathrm{mM} \mathrm{2}$ ) and $1 \%(0.1 \mathrm{mM})(25 \mathrm{mM} 1$ and $10 \mathrm{mM} 2)$, respectively, within $90 \mathrm{~min}$ of reaction (Table 1).

The high sensitivity was comparable to that recently report in the diamine assay (Scheme 1B). ${ }^{12}$ However, the colour intensity and levels of conversion also had a good correlation at conversions

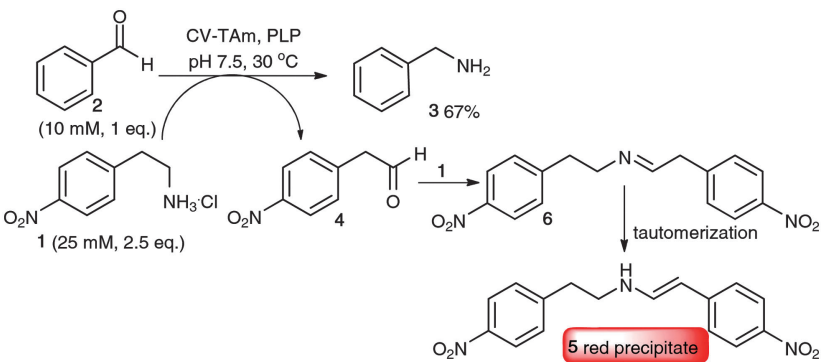

Scheme 2 Use of 2-(4-nitrophenyl)ethan-1-amine 1 as amine donor in a transamination reaction with CV-TAm $\left(0.4 \mathrm{mg}\right.$ protein $\left.\mathrm{mL}^{-1}\right)$.

Table 1 Study to establish the sensitivity of the colorimetric assay using 1. Reactions were performed using CV-TAm clarified cell lysate and different ratios of $\mathbf{1}$ and acceptor benzaldehyde $\mathbf{2}^{a}$

\begin{tabular}{lllll}
$\begin{array}{llll}\text { Cell lysate } \\
\text { conc. }^{b}\end{array}$ & \multicolumn{1}{c}{$\mathbf{1}(25 \mathrm{mM})$ and $2(10 \mathrm{mM})$} & & $\mathbf{1}(12.5 \mathrm{mM})$ and $2(5 \mathrm{mM})$ \\
\cline { 2 - 3 } Conversion $^{c}$ & Coloration & & Conversion $^{c}$ & Coloration \\
\hline 0.004 & 0.5 & 1.5 \\
0.01 & 0.5 & 1.5 \\
0.02 & 1.0 & 2.0 \\
0.06 & 1.5 & 3.5 \\
0.10 & 3.5 & 6.5 \\
0.14 & 5.5 & 9.0 \\
0.18 & 6.0 & 12.5 \\
0.22 & 6.5 & 14.0 \\
0.26 & 7.5 & 16.5 \\
0.30 & 9.5 & 19.5 \\
0.40 & 16.0 & 28.5 \\
0.50 & 21.0 & & 34.5
\end{tabular}

${ }^{a}$ Reactions were performed in triplicate. ${ }^{b} \mathrm{In} \mathrm{mg}$ protein $\mathrm{mL}^{-1}$. ${ }^{c}$ Calculated based on the formation of 3 by HPLC $(210 \mathrm{~nm})$.

of up to $\sim 30 \%$. As conversion yields can be directly estimated in this range based on the colour intensity of the precipitate, this assay importantly offers a simple and sensitive screening approach to determine TAm activity. It was also confirmed that the assay could be performed in the $\mathrm{pH}$ range of $\mathrm{pH} 6.0$ to 9.0.

To demonstrate the versatility of the colorimetric TAm screening method, reactions with $(S)$-selective $\omega$-TAms CV-TAm, Pseudomonas putida PP_0596 (Pp-TAm), ${ }^{7 g}$ and KPN00799 Klebsiella pneumoniae KPN_00799 (Kp-TAm), ${ }^{16}$ as well as ArRMut11, an $(R)$-selective amine transaminase variant that was generated for the amination of sterically hindered ketones, ${ }^{7 a}$ were performed. Together with the amine donor $\mathbf{1}(25 \mathrm{mM})$ aldehydes, 2, butyraldehyde 7 , and cyclohexane carboxaldehyde $\mathbf{8}$, and ketones, acetophenone 9, 2-butanone 10, and cyclohexanone 11, were used as amine acceptors $(10 \mathrm{mM})$ (Fig. 1$).$ Control reactions with an amino acceptor but lacking cell lysate remained as a pale yellow coloration after $18 \mathrm{~h}$ of reaction with 1 (E1-E6). Bioconversion control reactions without an amino acceptor but with cell lysate resulted in a slight yellow coloration with CV-TAm and Pp-TAm (A7, B7), presumably because of the background conversion of enzyme-bound PLP to PMP, ${ }^{17}$ whereas control bioconversions using Kp-TAm and ArRMut11 showed no background coloration. For each enzyme, background activity resulted in significantly less coloration than observed during the reaction with the amine acceptor. In general, significant colour changes were observed 


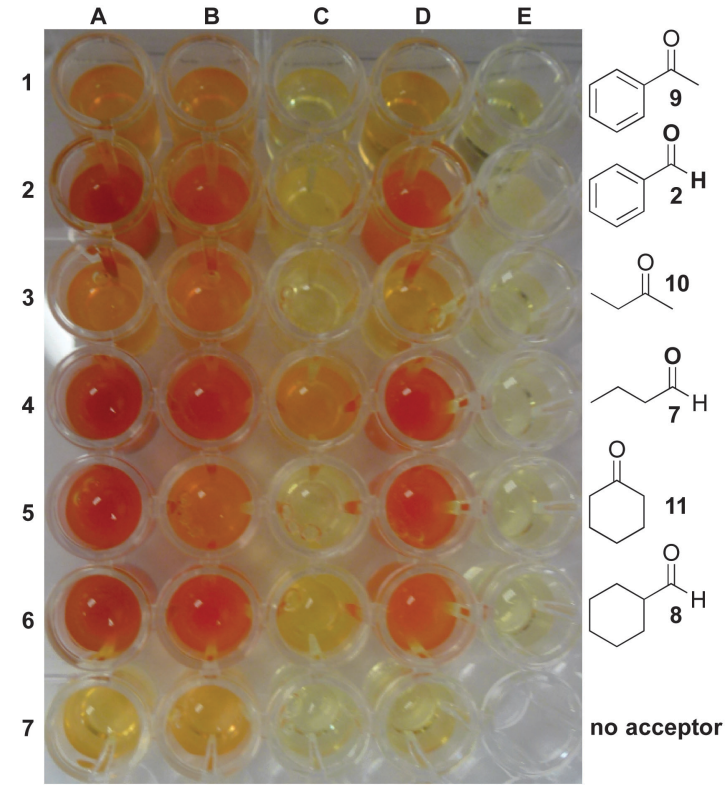

Fig. 1 2-(4-Nitrophenyl)ethan-1-amine 1 based transaminase screening. The assay was performed in triplicate. $1(25 \mathrm{mM})$, amino acceptor $(10 \mathrm{mM})$, PLP (1 mM), KPi buffer pH 7.5 (100 mM) and enzyme as crude lysate, $18 \mathrm{~h}$, $30{ }^{\circ} \mathrm{C}, 200 \mathrm{rpm}$. (A) CV-TAm; (B) Pp-TAm; (C) Kp-TAm; (D) ArRMut11; (E) no enzyme.

with most enzymes in bioconversions (A-D: 1-6). CV-TAm, for example, readily accepted amine acceptors $2,7,8$ and 11 (A2, A4-A6) resulting in intensely red coloured solutions. However, with amine acceptors $\mathbf{9}$ and $\mathbf{1 0}$ substantially less coloration was observed (A1, A3) indicating only moderate conversion of these substrates.

Bioconversions with Pp-TAm and ArRMut11 gave similar results, however, with less intense coloration (B1-B6, D1-D6) in particular with amine acceptor $\mathbf{1 1}$ (B5, D5) compared to the CV-TAm reactions. Amongst all enzymes tested, Kp-TAm showed only moderate acceptance of the different aldehydes and ketones under the reaction conditions used, as indicated by the slight coloration with amine acceptors 2 (C2), 7 (C4) and 10 (C6) and no colour change with 9 (C1), 10 (C3) and $\mathbf{1 1}$ (C5). To confirm the reliability of this assay, the conversion of the acceptors into the corresponding amines was determined by HPLC analysis and a good correlation was observed (see ESI $\dagger$ ). For example, bioconversions with CV-TAm, Pp-TAm and ArRMut11 resulted in low but detectable levels of colour change with amine acceptors 9 (A1, B1, D1) and 10 (A3, B3, D3), which proceeded with moderate conversions of $1-4 \%$. Combined, these results clearly demonstrated that the colorimetric assay developed offers a simple, rapid and sensitive HT platform for the evaluation and substrate profiling of large enzyme libraries. For lower conversions and lower substrate concentrations, these can be determined quantitatively.

Since the 4-nitroaryl electron withdrawing group (EWG) in $\mathbf{5}$ will enhance the tendency for it to form an enamine, other commercially available amine donors possessing EWGs were investigated to establish the wider generality of the assay: 4-(2aminoethyl)benzonitrile hydrochloride $\mathbf{1 2}$, and a cyclic analogue of 1, 5-nitro-2,3-dihydro- $1 H$-inden-2-amine hydrochloride 13,

were used. Computational docking of the amino donors, and with PLP intermediates, into the active site of CV-TAm crystal structure (PDB ID: 4AH3) using Autodock Vina ${ }^{18}$ confirmed that both analogues can readily gain access to the active site with close proximity to PLP (ESI $\dagger$ Fig. S1). Assays were performed with the amine acceptor 7, which led to the formation of a yellow and brownish coloured precipitate, with $\mathbf{1 2}$ and $\mathbf{1 3}$ respectively (Fig. 2). Butylamine formation was confirmed by HPLC analysis showing that reactions proceeded with similar conversions to those observed with amine donor 1, 74\% (with 12) and 65\% (with 13). Due to the formation of a yellow precipitate, which is the same colour as PLP, compound $\mathbf{1 2}$ is less suited as an amino donor. However, amine donor $\mathbf{1 3}$ can also be used for the screening of TAms, and as a cyclic donor may be useful to identify TAms that are able to accept cyclic substrates.

Apart from the application in multi-well plates, a colony-based colorimetric assay to provide a HT method that is amendable for rapid screening of TAm variant libraries was also developed. In a control reaction with wild type E. coli BL21 (DE3) incubated with 1 $(12.5 \mathrm{mM})$ and $2(5 \mathrm{mM})$, background conversion by the host intrinsic enzymes was excluded as it showed no coloration (Fig. 3A). However, the conversion of $1(12.5 \mathrm{mM})$ and $2(5 \mathrm{mM})$ with recombinant $E$. coli BL21 (DE3) containing CV-TAm resulted in the formation of intensely red coloured colonies (Fig. 3B). In contrast, control reactions without amine acceptor 2 led to the formation of faintly orange colonies (Fig. 3C) due to background conversions with residual intracellular acceptors such as pyruvate. However, a clearly visible difference in colour intensity was observed. Compared to previously published solid-phase TAm screening methods ${ }^{12,15}$ this assay used a single amine donor to identify TAm activity and moreover differentiates between enzyme activity with a target substrate and residual activity with intracellular acceptors.

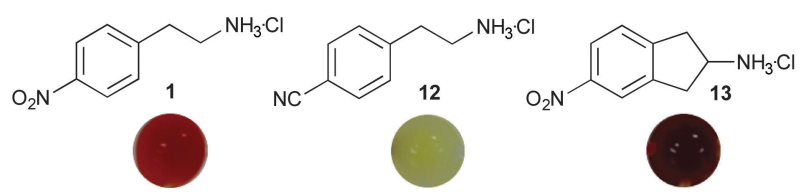

Fig. 2 Assay coloration when using amino donors 1, 12 and 13 with CV-TAm and acceptor 7.

A

Fig. 3 Colony-based TAm screening assay using amine donor 1 (12.5 mM) and acceptor benzaldehyde $2(5 \mathrm{mM})$ at $30{ }^{\circ} \mathrm{C}$ for $30 \mathrm{~min}$. The assay was performed in triplicate. Control assay with WT E. coli BL21 (DE3) with 2 (A). Assays using E. coli BL21 (DE3) containing CV-TAm with 2 (B) and without 2 (C). 
In summary, we have developed a sensitive colorimetric assay that enables the rapid and low cost screening and substrate profiling of $(S)$ - and $(R)$-selective TAms. The application of commercially available 2-(4-nitrophenyl)ethan-1-amine hydrochloride $\mathbf{1}$ also allows reaction monitoring by the level of intensity of a coloured precipitate. Furthermore, the use of other analogues such as 13, possessing an EWG at the C-2- or C-4 position on the aromatic ring of phenethylamine-based linear or cyclic amine donors, in TAm substrate-profiling screens is possible. In addition, the assay is amendable for the HT screening of large TAm libraries, including mutant libraries, against panels of acceptor substrates on solid-phase. This operationally simple screening method offers an ideal strategy to avoid expensive equipment requirements, and the time-consuming and technically demanding analysis of TAm reactions via HPLC, GC or spectrophotometrically, and has enormous potential in new TAm discovery and substrate profiling.

We gratefully acknowledge the Biotechnology and Biosciences Research Council (BBSRC) (BB/L007444/1) and Almac for funding D. B., and Novartis Pharmaceuticals for funding N. L.

\section{Notes and references}

\$ Typical screening procedure: the transaminase enzymatic reaction was performed in 96 well-plate with a total volume of $200 \mu \mathrm{L}$ containing 2-(4nitrophenyl)ethan-1-amine hydrochloride $1(25 \mathrm{mM})$ as amine donor, an aldehyde or a ketone $(10 \mathrm{mM})$ as amine acceptor, PLP $(0.2 \mathrm{mM})$, potassium phosphate buffer $\mathrm{pH} 6.0$ to $\mathrm{pH} 9.0(100 \mathrm{mM})$, TAm cell lysate $\left(0.4 \mathrm{mg} \mathrm{mL}{ }^{-1}\right)$ at $30{ }^{\circ} \mathrm{C}$ and $500 \mathrm{rpm}$ for 18 hours. The reaction was started by the addition of amine donor 1 . Two negative controls were also performed, one without aldehyde or ketone and another without enzyme. Transaminases active towards the selected aldehydes or ketones were indicated by the orange/red coloration (Table 1 and Fig. 1).

1 (a) M. Höhne and U. T. Bornscheuer, ChemCatChem, 2009, 1, 42; (b) H. Kohls, F. Steffen-Munsberg and M. Höhne, Curr. Opin. Chem. Biol., 2014, 19, 180; (c) R. C. Simon, N. Richter, E. Busto and W. Kroutil, ACS Catal., 2014, 4, 129; (d) E. O'Reilly and N. J. Turner, Perspect. Psychol. Sci., 2015, 4, 55.

2 (a) N. Uematsu, A. Fujii, S. Hashiguchi, T. Ikariya and R. Noyori, J. Am. Chem. Soc., 1996, 118, 4916; (b) T. C. Nugent and M. El-Shazly, Adv. Synth. Catal., 2010, 352, 753; (c) D. Ghislieri and N. J. Turner, Top. Catal., 2013, 57, 284.

3 T. C. Nugent, Chiral Amine Synthesis, Wiley-VCH Verlag GmbH \& Co. KGaA, Weinheim, Germany, 2010.
4 J. Ward and R. Wohlgemuth, Curr. Org. Chem., 2010, 14, 1914.

5 For reviews: (a) D. Koszelewski, K. Tauber, K. Faber and W. Kroutil, Trends Biotechnol., 2010, 28, 324; (b) M. S. Malik, E. S. Park and J. S. Shin, Appl. Microbiol. Biotechnol., 2012, 94, 1163; (c) S. Mathew and H. Yun, ACS Catal., 2012, 2, 993.

6 For example: (a) K. E. Cassimjee, C. Branneby, V. Abedi, A. Wells and P. Berglund, Chem. Commun., 2010, 46, 5569; (b) A. Cuetos, I. Lavendera and V. Gotor, Chem. Commun., 2013, 49, 10688; (c) C. E. Paul, M. Rodríguez-Mata, E. Busto, I. Lavendera, V. GotorFernández, V. Gotor, S. García-Cerrada, J. Mendiola, O. de Frutos and I. Collado, Org. Process Res. Dev., 2014, 18, 788; (d) C. Sayer, R. J. Martinez-Torres, N. Richter, M. N. Isupov, H. C. Hailes, J. Littlechild and J. M. Ward, FEBS J., 2014, 281, 2240; (e) E. O'Reilly, C. Iglesias, D. Ghislieri, J. Hopwood, J. L. Galman, R. C. Lloyd and N. J. Turner, Angew. Chem., Int. Ed., 2014, 53, 2447.

7 For example: (a) C. K. Savile, J. M. Janey, E. C. Mundorff, J. C. Moore, S. Tam, W. R. Jarvis, J. C. Colbeck, A. Krebber, F. J. Fleitz, J. Brands, P. N. Devine, G. W. Huisman and G. J. Hughes, Science, 2010, 329, 305; (b) T. Sehl, H. C. Hailes, J. M. Ward, R. Wardenga, E. von Lieres, H. Offermann, R. Westphal, M. Pohl and D. Rother, Angew. Chem., Int. Ed., 2013, 52, 6772; (c) N. Richter, R. C. Simon, W. Kroutil, J. M. Ward and H. C. Hailes, Chem. Commun., 2014, 50, 6098; (d) J. Limanto, E. R. Ashley, J. Yin, G. L. Beutner, B. T. Grau, A. M. Kassim, M. M. Kim, A. Klapers, Z. Liu, H. R. Strotman and M. D. Truppo, Org. Lett., 2014, 16, 2716; (e) C. K. Chung, P. G. Bulger, B. Kosjek, K. M. Belyk, N. Rivera, M. E. Scott, G. R. Humphrey, J. Limanto, D. C. Bachert and K. M. Emerson, Org. Process Res. Dev., 2014, 18, 215; $(f)$ E. Busto, R. C. Simon, B. Grischek, V. GotorFernandez and W. Kroutil, Adv. Synth. Catal., 2014, 356, 1937; (g) B. R. Lichman, E. D. Lamming, T. Pesnot, J. M. Smith, H. C. Hailes and J. M. Ward, Green Chem., 2015, 17, 852.

8 S. Mathew, G. Shin, M. Shon and H. Yun, Biotechnol. Bioprocess Eng., 2013, 18, 1.

9 U. Kaulmann, K. Smithies, M. E. B. Smith, H. C. Hailes and J. M. Ward, Enzyme Microb. Technol., 2007, 41, 628.

10 S. Schätzle, M. Höhne, E. Redestad, K. Robins and U. T. Bornscheuer, Anal. Chem., 2009, 81, 8244.

11 M. D. Truppo, J. D. Rozzell, J. C. Moore and N. J. Turner, Org. Biomol. Chem., 2009, 7, 395.

12 A. P. Green, N. J. Turner and E. O'Reilly, Angew. Chem., Int. Ed., 2014, 53, 10714.

13 B.-Y. Hwang and B.-G. Kim, Enzyme Microb. Technol., 2004, 34, 429.

14 J. Hopwood, M. D. Truppo, N. J. Turner and R. C. Lloyd, Chem. Commun., 2011, 47, 773.

15 M. S. Weiß, I. V Pavlidis, C. Vickers, M. Höhne and U. T. Bornscheuer, Anal. Chem., 2014, 86, 11847.

16 (a) N. Richter, R. C. Simon, H. Lechner, W. Kroutil, J. M. Ward and H. C. Hailes, Org. Biomol. Chem., 2015, 13, 8843; (b) R. J. Martinez-Torres, A. Bour, I. N. Taylor, H. C. Hailes and J. M. Ward, in preparation.

17 U. Schell, R. Wohlgemuth and J. M. Ward, J. Mol. Catal. B: Enzym., 2009, 59, 279.

18 O. Trott and A. J. Olson, J. Comput. Chem., 2010, 31, 455. 